\title{
Agnostic Biosignature Exploration at Europa through Plume Sampling
}

A White Paper submitted to the Decadal Survey in Planetary Science and Astrobiology

Paul Mahaffy, Solar System Exploration Division/Code 690,

NASA Goddard Space Flight Center, 8800 Greenbelt Rd, Greenbelt, MD 20771

Paul.R.Mahaffy@nasa.gov

\section{Co-Authors:}

Margaret Weng, Georgetown University, Ricardo Arevalo, University of Maryland Mehdi Benna, NASA Goddard and University of Maryland, Roger Summons, MIT, Will Brinckerhoff, NASA Goddard, Morgan Cable, Jet Propulsion Laboratory, Kathleen Craft, Johns Hopkins APL, Jen Eigenbrode, NASA Goddard,

Bill Farrell, NASA Goddard, James Garvin, NASA Goddard,

John Grunsfeld, Endless Frontier Associates LLC, Alexis Templeton, University of Colordo, Sarah Johnson, Georgetown University, Tori Hoehler, NASA Ames

Alison Murray, Desert Research Institute, William Sparks, SETI Institute

\section{Co-Signers:}

Maëva Millan, NASA Goddard and Georgetown University, Heather Graham, NASA Goddard, Luoth Chou, NASA Goddard and Georgetown University, Victoria Da Poian, NASA Goddard, Natalie Grefenstette, Santa Fe Institute \& Blue Marble Space Institute of Science

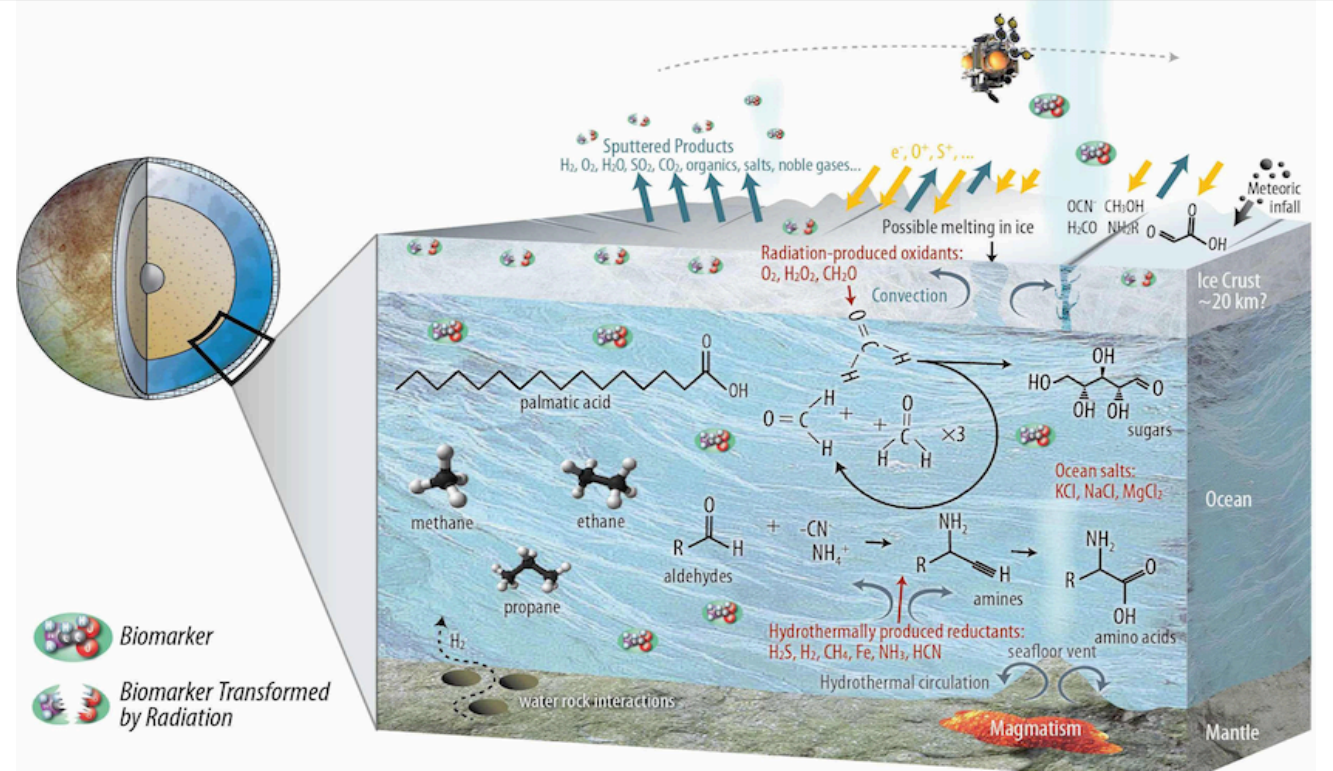

Water-rock interactions, potentially enhanced by tidal heating, could supply the Europan ocean with chemical energy sources and material resources required by active life. An Astrobiology Biosignature Explorer at Europa (ABEE) New Frontier mission would be designed to search for biomolecules contained in plume material. 
The search for the presence and history of life in the universe is one of the core focus areas of NASA. Within our solar system, the icy moons of Jupiter and Saturn provide compelling targets for a detailed search for microbial life. Abundant water, rock/water interfaces, and a variety of chemical energy sources may have provided the conditions for life to emerge and persist. Potential life activity may be sustained by hydrogen or methane derived from water/rock reactions between the ocean and crust coupled with oxidants such as sulfate or $\mathrm{CO}_{2}$ delivered to the ocean from overturn of the exterior ice surface. A focused New Frontier mission with in situ sampling at icy moon targets could be designed to reveal the detailed structure, complexity, stereochemistry, and distribution of organic compounds that may provide definitive evidence for the presence of biology even if the molecular basis of this life is distinct from that on Earth.

To cast the widest net for life detection, it is essential to utilize agnostic biosignature detection methods that do not assume that extraterrestrial life has followed the same chemical evolution pathway as terrestrial life. A detection of extant life elsewhere in our solar system would have profound implications for the possibility of widespread life in the universe. Thus, a systematic approach to the ambitious task of searching for biosignatures at the icy moons of Jupiter and Saturn deserves careful consideration by the Decadal Survey in Planetary Science and Astrobiology. The Agnostic Biosignature Explorer at Europa (ABEE) mission would be designed to collect and analyze plume material at Europa to search for molecular evidence of life. ABEE mission architecture would complement the Clipper mission, a future Europa Lander mission, and Enceladus plume missions.

The search for life at ocean worlds is supported by both political and scientific leadership. A congressional appropriation bill (H. Rept. 115-704, 2019) recently called for implementation of an ocean worlds program whose primary goal "is to discover extant life on another planet using a mix of Discovery, New Frontiers and flagship or strategic missions." Around the same time, a report from the National Academies of Sciences, Engineering, and Medicine entitled An Astrobiology Strategy for the Search for Life in the Universe (Nat. Acad., 2019) provides specific direction to NASA to infuse astrobiology objectives and an expanded search for extraterrestrial life into its exploration program. However, these ambitious goals can only be met by the synergistic alignment of multiple missions - no one proposed payload can successfully investigate all relevant scientific questions. The Europa Clipper orbital mission, currently under development, is designed to examine Europa's potential habitability but with no requirement to conduct a search for life. The ongoing study of a short-lived Europa lander mission is more firmly directed toward biosignature detection. NASA considered concepts for adding detachable payloads to the Europa Clipper mission to fly through plumes and conduct a more direct search for life, but these were constrained by available resources on the host Clipper spacecraft and abandoned by NASA in 2016. The ABEE would provide an optimal approach to discovery of extraterrestrial life in icy moon ocean worlds by leveraging concepts from Europa Clipper, Lander and Enceladus plume flythrough studies. The ABEE employs a strategy customized to the specific challenges of the plume environment at Europa and would employ the latest advances toward space qualified instrumentation for agnostic biosignature analyses (see Universal Life Detection white paper, lead authors Chou \& Grefenstette).

Approach to Life Detection with Europa Plume Sampling:

1. Evidence for Plumes at Europa and Cryogenic Activity: Material from the subsurface ocean of Europa, including biomolecules, may be transported to the surface and provide targets for life detection. These subsurface materials would be detectable as water vapor plumes or cryovolcanism signatures. There are currently four independent observation types that imply the 
presence of plumes on Europa (emission line imaging, continuum absorption imaging, ionospheric profile analysis, and in situ plasma measurements), and correlations between these and an independent dataset (thermal anomaly). Detections of intermittent excesses of Lyman $\alpha$ and oxygen OI $130.4 \mathrm{~nm}$ emission beyond the limb of Europa using the Hubble Space Telescope (HST) ultraviolet emission-line images (Figure 1) are indicative of water vapor plumes near the southern polar regions (Roth et al. $2014 \mathrm{a}, \mathrm{b}$ ). These detections were further supported by Sparks et al. (2016), who used the Hubble Space Telescope in a high resolution far ultraviolet imaging mode, to seek exospheric continuum absorption from gas or aerosols associated with plumes as Europa passed in front of the smooth face of Jupiter. Three candidate absorption features were identified, one near the Pwyll crater, closer to the equator than the previously seen candidates. These plume candidates were of a height and implied column density similar to those of Roth et al. (2014a). The plume described on March 17, 2014 by Sparks et al. (2016) was found to repeat in an identical location two years later. This information is critical in establishing the veracity and timescale of a variable or intermittent phenomenon and raised the possibility of a consistently active source of erupting material on Europa. This conclusion was bolstered by comparison with a nighttime thermal image from the Galileo Photopolarimeter-Radiometer (PPR) which showed a thermal anomaly at the same location (Spencer et al. 1999). The anomaly has the highest observed brightness temperature on the Europa nightside.

These findings

also suggest intriguing differentiation of the local surface from its surroundings. If the thermal anomaly is due to an internal heat source, subsurface liquid water at depth $\sim 2 \mathrm{~km}$ would be suggested, consistent with scenarios in which a liquid water reservoir has formed within a thick ice shell. However, the
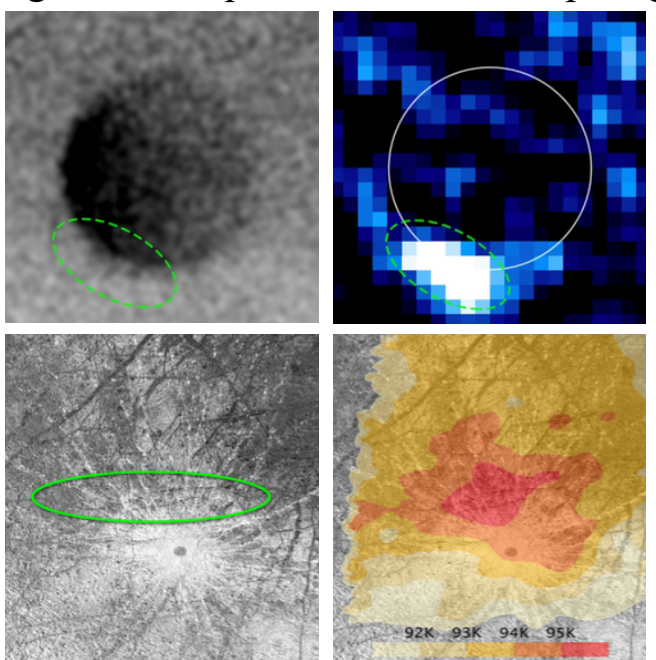

Figure 1. Plume candidates in approximately the same location from (top left) transit continuum absorption image Jan. 2014 from Sparks et al. (2016) and (top right) a far ultraviolet emission image of Roth et al. (2014a). Mapped on Galileo/Voyager USGS image (bottom left). Contours of nighttime brightness temperature from Galileo PPR instrument (bottom right) from Spencer et al. (1999). This thermal anomaly is the warmest point of the observed Europa night side coincident with the location of the repeated plume candidate.

currently favored interpretation of the Galileo thermal data coupled to ALMA observations, is that the region has higher thermal inertia than its surroundings (Trumbo et al. 2017; 2018). Hence, surface conditions in this region differ, potentially due to deposition from a plume ejecta blanket, or to surface disturbance and disruption.

Additional in situ measurements from the Galileo spacecraft have been identified which provide independent support for the presence of plumes. The repeat-plume imaging detection near Pwyll was found to spatially coincide with the Galileo radio occultation E6an entrance profile (McGrath \& Sparks 2017). Jia et al. (2018) reported on magnetic field and plasma wave observations acquired on Galileo's closest encounter with the moon as in-situ evidence of a plume on Europa, and Huybrighs et al. (2020) inferred the presence of a plume from energetic proton depletions. The location, duration and variations of the magnetic field and plasma wave measurements were consistent with the interaction of Jupiter's corotating plasma with Europa if a plume with characteristics inferred from Hubble images was erupting from a region relatively close to Europa's Pwyll thermal anomaly at the time of the Galileo flyby. 


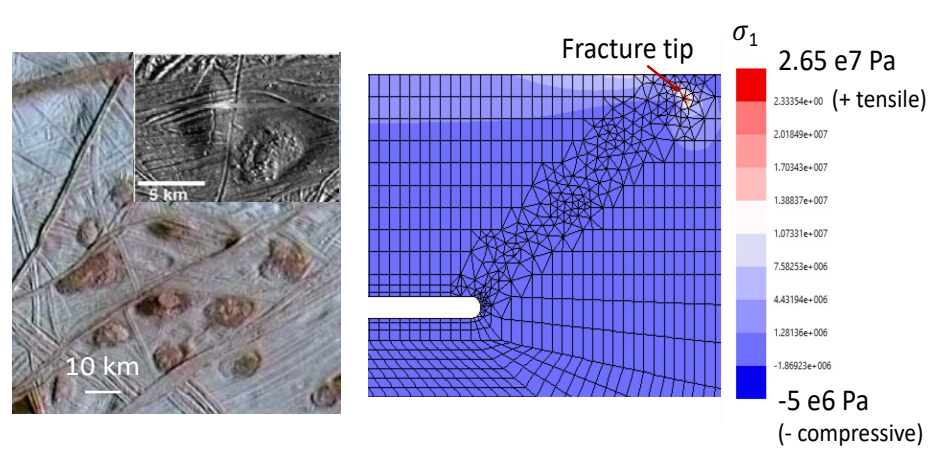

Figure 2. Fluid transport toward the surface illustrated by pits and domes on Europa (left) and model showing fracture (right) propagated up from a subsurface, over pressurized sill $\sim 1$ km deep (Craft et al., 2019a)
The demonstrated evidence and multiple detections of plumes is encouraging for finding ocean signatures in the exosphere. Plume sources are likely liquid reservoirs located near the surface in Europa's ice shell. These liquid pockets would produce temperature anomalies that would last $\sim 1000$ years and persist even if the liquid is cut off from the heat source (Craft et al., 2016) and may allow for periodic eruptions over this entire time. The persistence of thermal signatures is a straight-

forward method of identifying the plume source regions. If Europa was active between 1997 (Jia et al. 2018) and 2012 (Roth et al 2014a) or 2016 (Sparks et al. 2018), it could still be active when ABEE arrives.

Cryovolcanism on Europa may be bringing fluids to ortoward the surface ice. In addition to silicate-based magma, volcanism has also been postulated to occur in icy bodies, with a warm, salty, water-ice mixture for the magma (e.g. Fagents, 2003; Kargel, 1991). Magma surface extrusion forms raised features and salt deposits, such as the pits and domes on Europa and the Ahuna Mons dome on Ceres (Figure 3). Overpressurization could have driven fluids from a subsurface reservoir on Ceres (Neveu and Desch, 2015; Quick et al., 2017) but these processes may also occur on icy moons (e.g. Fagents, 2003; Walker and Schmidt, 2018). Work by Craft et al. (2019a) showed how a pressure increase in a cryovolcanic chamber in Europa's subsurface, caused by cooling and freezing of the fluid, could initiate and drive dike-like fractures to the surface. Cryomagma from the sill could be transported with the advancing dike and the formation of bright deposits and/or deformation of the surface could occur.

2. Agnostic Life Detection Measurements: Flythrough plume sampling offers a straightforward way to access subsurface fluids and any biomolecules while minimizing radiation exposure. Such sampling should be coupled with agnostic methods of life detection, which are an exciting new frontier of current research. These methods are particularly relevant to ocean worlds, where environmental conditions for life's origin and subsequent persistence may be substantially different than that of Earth. They are also supported by high heritage equipment such as mass spectrometry, making these approaches adaptable and relevant for current and future missions. The ABEE mission would utilize agnostic techniques to maximize the life detection capabilities for plume samples. Molecular biosignatures may be present in the complex mix of compounds dissolved in ocean waters or as particulate matter that makes its way from the Europa ocean into a plume. These are highly compelling agnostic targets, as carbon-containing molecules may exhibit patterns of molecular weight, stereochemistry, or isomerization (Summons, et al., 2008) that distinguish them from carbonaceous material produced in the interstellar medium which had been delivered to Europa. Chemical analysis of these compounds can be independent of the particular set of signatures that terrestrial life produces and simply need to reveal the existence of patterns or enantiomeric enhancements that are absent from abiogenic mixtures. Such patterns can distinguish between molecules that have been constructed systematically from small universal precursors versus those constructed randomly. Some of these signatures present 
in terrestrial aqueous or mineral samples are illustrated in Figure 3. Separation techniques such as gas or liquid chromatography or capillary electrophoresis combined with mass spectrometers provide powerful tools to conduct these investigations.

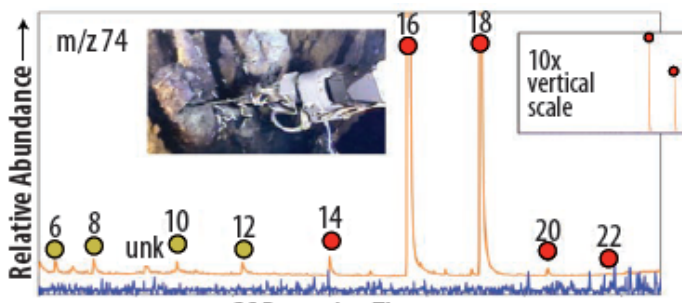

GC Retention Time $\longrightarrow$

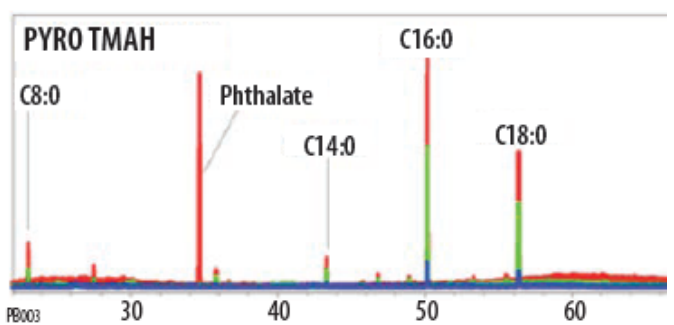

Figure 3. The biosignatures evident in these chromatograms are the patterns in carbon chain length of lipids extracted from both fluids and rocks in NASA Goddard labs with GCMS. A single mass chromatogram (left) showing trace concentrations of lipids extracted from less than 5 microliters of a water sample from seawater adjacent to the Rainbow hydrothermal vent processed with tetramethylammonium hydroxide (TMAH) and a chromatogram (right) of lipids extracted from recently extant life in iron oxides (different colors show mass values 74,87 , and $143 \mathrm{Da})$.

One focus of the Laboratory for Agnostic Biosignatures, supported by NASA under the Network for Life Detection (NfoLD) coordination network, is to evaluate the hypothesis that the chemical complexity of a compound can in itself point to an origin from biotic or abiotic processes. A complexity measure designated 'pathway complexity' based on graph theory and the minimum number of possible paths to construct a molecule has been developed (Marshall et al., 2017). One way to experimentally interrogate the structural complexity of a high molecular weight organic compound is to isolate it in the analyzer of a flight-capable ion trap mass spectrometer and then create fragments using the tandem mass spectrometry capability of these instruments. For high molecular weight molecules, analysis of the fragment ions selectively generated from specific molecular ions, or higher order fragment ions, can provide a layer of information about structural details that might not be evident in a conventional mass spectrum. Molecular approaches for agnostic life detection and the instrumentation and advancement of the maturity of the related technologies for space flight applications are active areas of research and have been highlighted by the recent Europa Lander Science Definition Team (Hand et al., 2017). 3. Relevant Analog Environments: The abundance and productivity of Europan life, if present, may be diminished relative to the well-studied environments at Earth's surface. Analog lowenergy or low-biomass sites on Earth useful for studies of Europa include permanently icecovered Antarctic lakes, the extremely lean waters of deep Atlantic bottom water (Meyer at al., 2016) Earth's deep fracture fluids (Lin et al., 2006; Hollard et al., 2013), and subsurface fluids rich in sulfate and reductants, like the Borup Pass in the Canadian High Arctic, where hydrogen sulfide-rich fluid is erupted each spring, forming a sulfidic icing that traps salts, organisms, and a diversity of organic byproducts (Grasby et al., 2004; Gleeson et al., 2011; Lau et al., 2017).

Building an Agnostic Biosignature Explorer at Europa: Key Considerations 1. Range of Measurements and Candidate Instruments for Life Detection: To enable agnostic life detection, a suite of instruments to take organic inventory, including abundance distributions, molecular patterns, isotopic composition, structural affinity, and isomerization, should be prioritized with corroborative and/or redundant proxies for statistical robustness.

In contrast to the Clipper payload, separation techniques such as gas chromatography (GC), liquid chromatography (LC), and/or capillary electrophoresis (CE), are critically needed for a life detection mission to: i) isolate and quantify different classes of organic 
compounds; ii) enhance sensitivity; iii) distinguish structural, geometrical, and optical isomers; iv) quantify enantiomeric excesses; and, v) distinguish potential isobaric interferences. For example, the SAM (Mahaffy et al., 2012) and MOMA instruments (Goesmann et al., 2017) utilize derivatization (e.g. silylation or methylation) of polar molecules such as amino and carboxylic acids to make these molecules more volatile to improve their detectability by GCMS techniques. Alternatively, approaches can be developed to target lipids, which are the building blocks of cell membranes in Earth's oceans and spring and lake analog environments. For example, tetramethylammonium hydroxide (TMAH) methylation provides chemical processing that is effective for analysis of lipids. In addition, fatty acids, which are the degradation products of lipids, can be analyzed as methyl esters (FAMES), preserving distinct biosignatures (Figure 3).

Coupled with mass spectrometry (MS), separation methods facilitate an adaptive and highly versatile suite of investigations to constrain plume composition. For example, the MOMA (Goesmann et al., 2017) and DragonFly DraMS instruments include a Chirasil-Dex column with DMF-DMA derivatization, which preserves the asymmetrical center of chiral molecules and supports isolation of optical isomers and quantification of enantiomeric excess. To access more refractory organic materials such as insoluble macromolecular complexes, laser desorption (LD) techniques, matured by these investigations, may also be of service for ABEE.

The linear ion trap qualified for the MOMA investigation enables selective ion isolation/enrichment via Stored Waveform Inverse Fourier Transform (SWIFT), and tandem mass spectrometry $\left(\mathrm{MS}^{\mathrm{n}}\right)$, supporting the derivation of molecular structure and providing insights into chemical complexity. Time-of-flight analyzers, such as the COSAC instrument flown on the ROSETTA mission (Goesmann et al., 2007), offer extended mass ranges in excess of kDa. In the next decade a suite of next-generation mass analyzers capable of advanced performance, such as ultrahigh mass resolution $(\mathrm{m} / \Delta \mathrm{m}>100,000 \mathrm{FWHM}$; Arevalo et al., 2018), will become available at lower risk suitable for the ABEE mission.

2. Context Instrumentation: The ABEE platform will enable hyperspectral imaging of the plume source. High resolution (2-3 m/pixel for a $5 \mathrm{~km}$ flyby altitude) enables detailed stereo examination of tectonic, chaos, and flow surface features, to complement the Europa Clipper mission's Europa Imaging System (EIS) cameras whose highest resolution $(\sim 1 \mathrm{~m} / \mathrm{pixel})$ coverage is limited to small areas $(2 \mathrm{~km} \times 4 \mathrm{~km})$ in the sub/anti Jovian hemispheres. Robust polarimetric instrumentation, currently in development, can determine distributions of particle size, shape, and refractive indies through measurement of the polarization as a function of phase angle and wavelength.. Such an instrument can constrain surface compactness and offer a remote sensing tool for detection of chirality.

Likewise, a thermal camera could enable detection of hotspots associated with a plume that show a temperature contrast with the nearby terrain. Highly precise thermal measurements may not be necessary for their detection and accuracies of $\pm 5^{\circ} \mathrm{C}$ will allow the source of the plume material to be identified, the type of terrain associated with this activity, and a measure of the vent source temperature perhaps finding highly active regions by comparison to earlier thermal mapping from Europa Clipper.

3. Collection and Survival of Molecular Compounds: Collecting plume samples during flybys raises technical challenges inherent to the spacecraft speed at which the samples are captured. At flyby speeds of several $\mathrm{km} / \mathrm{sec}$, alteration of organic molecules by hypervelocity impact on the collector surfaces must be assessed and mitigated. Measurements made by Cassini/INMS during its many flybys of Enceladus demonstrated the survivability of small organics molecules (C1- 
C4) to unmitigated hypervelocity impacts on instrument surfaces at speed of up to $17 \mathrm{~km} / \mathrm{s}$ (Waite et al., 2009). However, laboratory measurements show that more complex organics molecules (such as amino acids and peptides) can withstand impact pressures of up to $15 \mathrm{GPa}$ (equivalent to impact speeds of $3 \mathrm{~km} / \mathrm{s}$ ) without noticeable alterations (Bertrand et al., 2009). At higher shock levels, partial racemization and chemical alteration may occur.

4. Sample Processing and Purification: Many challenges exist for in situ techniques searching for signatures of life in planetary environments including plume samples from ocean worlds. Collected samples are likely to be saline and contain very low biomass, if present. At Europa, there is the additional challenge of a strong radiation environment that could act to alter the structure of any biology present in a sample. A sample preparation process to purify samples, including separating out salts, while retaining amino acids and other biosignatures of interest is being developed on a microfluidic chip by Craft et al. (2019b). This technique can be used as a purification tool for multiple downstream biosignature detection analyses and is provided in a small, low mass and power package. In addition to purification, the sample preparation may provide a way to concentrate samples if signals of biomass exist below limits of detection.

5. Plume Characteristics: The launch velocity of plume material at Europa is similar to that at Enceladus (being fundamentally set by the thermodynamic properties of water not too far from the freezing point) but the subsequent dynamics are rather different due to Europa's larger gravity (e.g. Lorenz, 2016; Quick et al., 2013). Thus, the plume does not lose material at the escape velocity into space as on Enceladus, but rather, like the much more visible sulfurous plumes on Io (Lorenz, 2015), forms a fountain that rains back onto the surface. Quick et al. (2013) models suggest plume heights of $\sim 2.5$ to $26 \mathrm{~km}$ and recent HST observations indicate material can reach $\sim 200 \mathrm{~km}$ (Roth et al., 2014a). Some material is abstracted from the plume by electrodynamic forces. Models show (Lorenz, 2016) bacterial cells of 10 microns could be lofted even to the plume tops, but no particles larger than $2 \mathrm{~mm}$ are lofted above $2 \mathrm{~km}$. For example, for a predicted plume flux each $670 \mathrm{~cm}^{2}$ of collector surface exposed (BEE assumption) should intercept $\sim 10$ milligrams of material at $10 \mathrm{~km}$ altitude For a comparable $0.2 \mathrm{~m}^{2}$ collection area, cell counts can be extrapolated (Lorenz, 2016) for a range of analog terrestrial waters (Vostok $100 / \mathrm{ml}$ and Earth's ocean $\left.1 \times 10^{6} / \mathrm{ml}\right)$. Collector area and efficiency of both collection and transfer of material from the collector to the instruments must be considered to target at least sub parts per billion detection limits. Furthermore, both the plume's gas emission and particulate ejecta are injected into the Jovian magnetoplasma environment and will undergo physical and chemical changes upon interacting with the plasma. The plume gas and dust, which are considered independent, will become coupled via electrical processes. Ionization of plume gas (Johnson et al., 2006) and the subsequent negative charge of small particulates due to the absorption of free elections will cause acceleration and spatial shifting relative to the outflow direction of the larger grains in the plume due to the effects of the Lorentz electromagnetic forces acting on the grains (Farrell et al., 2012; Meier et al., 2014; Dong et al., 2015).Since much of the mass in the Enceladus plume is carried by the small particulates (Dong et al, 2015), their electrostatic migration away from the primary outflow direction will be considered in ABEE targeting of the Europa plume. The chemical modification of plume potential biosignatures due to the prevalent plasma low energy electrons must be considered as requirements for this mission are formulated.

6. Synergy with Ongoing and Future Europa Missions: ABEE could serve as a precursor for future landed Europa missions by informing whether a landing site near the plumes is the highest priority, relative to the detailed terrain observations from Clipper. If the presence 
of organics in the plumes is confirmed, landing sites near the vents would be high priority, while observations of low or no organics in a particular plume would prioritize sites elsewhere. ABEE's discoveries regarding molecular composition of plume material will inform what Europa Lander will be motivated to characterize in young ice selected for sampling. The sampling possibilities for ABEE may in fact be more promising than a landed mission, as plume material is directly reflective of subsurface ocean composition and has not been altered by longterm radiation exposure. The ABEE mission would not depend on success of the Europa Clipper habitability mission, yet it could make good use of its data and place any biosignature discoveries of ABEE in context. Key data from Europa Clipper will include: EIS camera imagery of the surface at high resolutions; Europa-Ultraviolet Spectrograph (Europa-UVS) data of atmospheric composition that could include plume vapor composition and vertical; the Radar for Europa Assessment and Sounding: Ocean to Near-surface (REASON) to indicate/detect subsurface water and brines from surface to $30 \mathrm{~km}$ depth; global Europa Thermal Emission Imaging System (E-THEMIS) temperature observations to characterize heat flow anomalies and regolith depths; magnetometry data indicative of the ocean salinity; radiation monitors; and the Mapping Imaging Spectrometer for Europa (MISE) instrument observations of the surface to characterize the surface materials and detect the distribution of compounds present. Additionally, in situ data collected by the dust, plasma, and mass spectrometer (SUrface Dust Mass Analyzer (SUDA), Plasma Instrument for Magnetic Sounding (PIMS) and Mass Spectrometer for Planetary Exploration (MASPEX) instruments will provide valuable information on exospheric composition and chemical constituents at Europa.

Future exploration of Europa (and other ocean worlds) by the Lander and beyond will eventually seek to reach the ocean and subsurface water pockets to directly sample for life. The Scientific Exploration Subsurface Access Mechanism for Europa (SESAME), has funded iceocean probe/cryobot development to reach the ocean and strategies and hardware development for communication from the probe to surface (Craft et al., 2019c). ABEE is synergistic with missions of these types as its instruments will enable sample handling and processing in a small volume workspace with power/mass constraints and with similar required autonomy as a small ice-ocean probe. Life detection and contextual instrumentation from ABEE and a Europa Lander will also pave the way for miniaturized instruments to fit within a cryobot at Europa.

Engineering Considerations. The Goddard team that developed the BEE proposal for the Europa Clipper has addressed a range of technical issues including radiation, planetary protection, contamination control, flyby velocity, number of flyby's, and a detailed analysis of plume targeting in the $5-10 \mathrm{~km}$ flyby altitude with UV imaging. This team will be glad to provide the Decadal panels with this detailed additional information on request

In conclusion, the ABEE mission serves myriad purposes in advancing the frontier of life detection and exploration in the outer solar system. First, it is part of an integrated mission framework designed to build upon science outcomes from previous missions while laying important groundwork for future exploration by a landed mission. Second, the ABEE will target active Europan plumes, exciting candidates for extant life detection due to their dynamic, potentially substrate-rich environment and the ABEE ability to sample "fresh" materials with organics minimally damaged by surface exposure to the severe radiation environment. Finally, this mission will approach plume sample chemical analysis from an agnostic perspective, greatly broadening our understanding of how to look for life "as we don't know it" as we probe farther into the mysterious and alien worlds of the outer solar system. 